\title{
MODELING STEERING USING THE QUEUEING NETWORK - MODEL HUMAN PROCESSOR (QN-MHP)
}

\author{
Omer Tsimhoni and Yili Liu \\ Department of Industrial and Operations Engineering \\ University of Michigan, Ann Arbor, Michigan
}

\begin{abstract}
The Queueing Network - Model Human Processor (QN-MHP) is a computational architecture that combines the mathematical theories and simulation methods of queueing networks $(\mathrm{QN})$ with the symbolic and procedure methods of a GOMS-style task description and the Model Human Processor (MHP). Using QN-MHP, a steering model was created to represent the concurrent perceptual, cognitive, and motor activities involved in vehicle steering as truly concurrent processes. The model was compared with driving performance of human subjects and demonstrated realistic steering behavior. It steered a simulated vehicle at a fixed speed within the lane boundaries of straight sections and curves of different radii. In a quantitative validation of several basic measures of driving performance, the steering model yielded steering angle and lateral position similar to the human subject data. This work showed the strength of QNMHP as a model of driving behavior. Ongoing work further develops the model by expanding the scope of the driving task and by adding a concurrent secondary in-vehicle task.
\end{abstract}

\section{INTRODUCTION}

Computational cognitive models can contribute considerably to driving-related human factors research. Computational models can make quantitative predictions for scenarios that have not been tested, and provide a precise common language for description of phenomena of interest. These models can simulate real-time driver performance and by detailing the underlying processes predict possible interference with secondary in-vehicle tasks. Integration of driving into an existing modeling architecture of cognitive performance widens the range of possible modeled tasks to include the range of tasks that architecture has already covered.

Levison (1993) developed a model called the integrated driver model to simulate driving performance while dialing and conversing on a cellular phone. His model combined two separate software modeling modules: a control theory based driver/vehicle module (Levison, 1989; Levison et al. 2001) and a custom made procedural module of in-vehicle tasks. It simulated continuous steering performance (at a fixed speed) as visual attention is diverted from the road to one or more monitoring locations associated with an in-vehicle task.

More recently, Salvucci, Boer, and Liu (2001) developed a driver performance model by integrating a cognitive modeling tool (ACT-R, Anderson and Lebiere, 1998) with a task analysis of vehicle control. Building on this driving model, Salvucci and Macuga (2002) developed a model of cell-phone dialing while driving. Their model predicted degraded steering performance when the driver was required to look away from the road. The main drawback of their model approach is the constraint to a serial line of cognitive processing. Parallel processes are interleaved into one serial process, resulting in its reliance on explicit and often taskspecific transfer of control between the concurrent tasks. A modeling architecture that is based on network rather than serial flow has the potential of solving this drawback.

To model concurrent activities in a truly concurrent manner and with one underlying context-free mental architecture rather than separate task specific modeling modules, our effort was directed toward modeling driving using a novel computational model, the QN-MHP (Queueing Network - Model Human Processor). The QN-MHP is a computational architecture that combines the mathematical theories and simulation methods of queueing networks with the symbolic and procedure methods of GOMS and the Model Human Processor (MHP). As a network architecture, queueing networks are particularly suited for modeling parallel activities and complex mental architectures. Symbolic models have particular strength in generating a person's actions in specific task situations. By integrating the two complementary approaches, the QN-MHP offers a modeling and simulation architecture for generating in real-time and mathematical modeling of parallel and complex activities. QN-MHP has been successfully used to model reaction time tasks and visual search tasks (Feyen and Liu, 2001a, 2001b).

In this paper, we describe our work of modeling the concurrent perceptual, cognitive, and motor activities involved in vehicle steering with the QN-MHP.

\section{METHOD}

\section{QN-MHP and ProModel}

QN-MHP is implemented in ProModel (ProModel solutions, version 2001), a simulation-based software that is widely used for manufacturing and operational applications, and provides a natural programming environment for queueing network simulation. In addition, it has built-in analysis tools and strong visualization capabilities. 
In QN-MHP, 20 servers represent different functional modules of the human perceptual, cognitive, and motor information processing system (Figure 1). (See Feyen and Liu, 2001a, for more details.) Stimuli enter the perceptual subnetwork carrying perceptual information, which is then processed by the cognitive subnetwork and converted into actions, carried out by the motor subnetwork. The front-end of the model is an MS-Excel file with data about the environment (stimuli and object description), the actuators and actions in use, parameters available in long-term memory, and a goal list based on a GOMS-style task description of the task. Flow of information entities through the network can be visualized to provide an assessment of the utility of servers and the progress of actions. An output data file, documenting overt actions (e.g., hand and eye movements) is produced for post-simulation analysis.
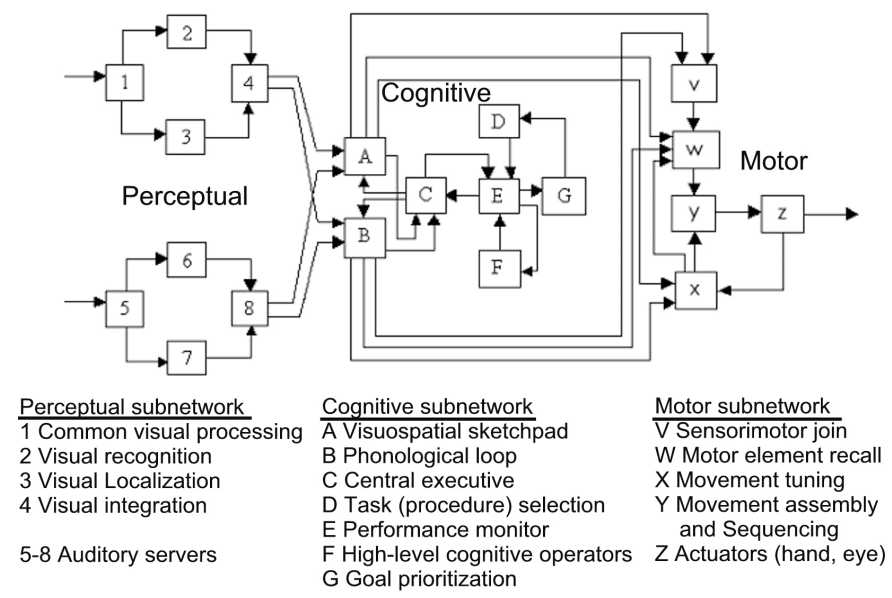

Figure 1. Layout of the servers in QN-MHP and the flow of information between them.

\section{Main Concepts of the Steering Model}

The steering model implemented in QN-MHP combines several concepts, as listed below.

Hierarchical task structure. Driving a vehicle can be described as a hierarchical combination of navigation, guidance, and vehicle control (McRuer, Allen, Weir, and Klein, 1977). It is for this reason that a top down, GOMSstyle task description, can provide a useful representation of driving. Following the hierarchical approach, the goal of steering the vehicle is a combination of subgoals for the perception of visual information about the vehicle, choice of steering strategy, and making the steering correction. It should be emphasized that these subgoals are accomplished concurrently as represented in QN-MHP, not serially. The magnitude of the target steering angle is calculated at a lower level of processing within the model and not at the task level.

Flow of visual input. In order to produce steering actions Drivers are generally thought to process splay angle (Loomis and Beal, 1998) and optical flow (Wann and Land, 2000). In the current model, inputs are available without actual image processing of a road scene, but the estimated time for such visual processing is taken into account in the visual servers as if they were processed. Consequently, the perceptual subnetwork provides vehicle lateral position, yaw angle, and curvature of oncoming curves after the processing time has passed. (The question of what exactly do drivers process in the driving scene is not the purpose of this model.)

Roles of focal and ambient visual systems. Research on visual guidance while driving (Leibowitz and Owens, 1977; Owens and Tyrell, 1999), has distinguished between the roles of the focal and ambient visual systems. A similar hypothesis was made by Mourant and Rockwell (1970) and further confirmed by Summala (1998). In the current model, most of the visual input for immediate steering is perceived by peripheral vision. Typical areas of visual input are in the lower periphery, around the lane markers and directly in front of the vehicle.

Near-far dichotomy. According to Donges (1978), steering is based on information from both near and far. This has also been addressed by Land and Lee (1994). The same notion of near and far is loosely followed in the current model. Visual input from lane markers and straight ahead at about $1 \mathrm{~s}$ in front of the vehicle is perceived peripherally and used for maintaining lane position. Visual input from greater distances (around 2-4 s down the road, and in curves, around the center of the lane at the point of tangency) is perceived foveally and used for determining curvature and heading changes.

Concurrent cognitive processing. The cognitive subnetwork of QN-MHP triggers eye movements towards desired stimuli in the road scene, sends the information for analysis, and based on the results, triggers motor actions. All these activities can occur concurrently in the network, so that consecutive processes do not have to wait for their predecessors to finish before they can start.

Steering movements. Hildreth, Beusmans, Boer, and Royden (2000) depicted lateral control as a set of basic sinelike steering wheel corrections with two phases. In a related study, Wallis, Chatziastros, and Bulthoff (2002) showed that when occluded, drivers completed only the first phase of such steering maneuvers, suggesting that the basic unit of steering is only half of a full sine wave. The second phase must be based on additional visual input. The current model utilizes single-phase open-loop corrections. A single-phase action of "steer-wait-steer back" is performed in an open-loop fashion, without visual input. However, before the second phase is performed, visual feedback is required.

Limited speed control. The current model steers the vehicle at a fixed speed of $72 \mathrm{~km} / \mathrm{hr}(45 \mathrm{mi} / \mathrm{hr})$. Speed control and the effects of road geometry on speed selection (e.g., Levison et al., 2002) add a level of complexity that is not simulated at this point.

No vestibular inputs. Vestibular inputs have considerable effects on speed adjustments, especially when driving on curves (Reymond et al., 2001). These effects are not considered in the current model. 


\section{Description of the Steering Model}

Table 1 provides a description of the steering model.

Table 1. Description of the steering model. (References to QN-MHP servers correspond to Figure 1)

\begin{tabular}{|c|c|}
\hline \multicolumn{2}{|l|}{ Inputs } \\
\hline Vehicle heading & $\begin{array}{l}\text { Vehicle heading relative to the road is retrieved as input to server } 1 \text { when fixating on a far point down the road, } \\
\text { about } 2-4 \mathrm{~s} \text { in front of the driver. }\end{array}$ \\
\hline Lateral position & $\begin{array}{l}\text { Lateral position relative to the center of the lane is retrieved as input to server } 1 \text { when one of three near points } \\
\text { (about } 1 \mathrm{~s} \text { in front of the driver, one at the lower center and two near each of the lane markers) is in the peripheral } \\
\text { vision of the driver. }\end{array}$ \\
\hline Road curvature & $\begin{array}{l}\text { On curves and approaches to curves, road curvature is retrieved as input to server } 1 \text { when the eye is fixated on a } \\
\text { far point about the point of tangency of the curve }\end{array}$ \\
\hline \multicolumn{2}{|r|}{ ( ) } \\
\hline Hand position & $\begin{array}{l}\text { The model outputs coordinates of hand position as the hand moves the steering wheel. (To interface with the } \\
\text { driving simulator, hand position is converted to corresponding steering angle by an intermediate module.) }\end{array}$ \\
\hline Eye position & Coordinates of the eye fixation point in space are output whenever the eye (server Z) is moved (by server $Y$ ). \\
\hline $\begin{array}{l}\text { Processing } \\
\text { Logic }\end{array}$ & $\begin{array}{l}\text { The main goal of maintaining the lane consists of subgoals for detecting the orientation parameters of the vehicle, } \\
\text { selecting a steering strategy, and steering the vehicle, correspondingly. }\end{array}$ \\
\hline $\begin{array}{l}\text { Detecting } \\
\text { orientation }\end{array}$ & $\begin{array}{l}\text { A 'watch for' cognitive command at server D directs the model's visual attention (Servers A and C issue } \\
\text { commands to and wait for proper types of entities from servers 1-4) to a far point when about to retrieve heading } \\
\text { and curvature and to a near point when about to retrieve lateral position. The eye is not moved if the near point } \\
\text { information is accessible from peripheral vision. Otherwise, the eye is moved to that point using a saccade. }\end{array}$ \\
\hline $\begin{array}{l}\text { Selecting a } \\
\text { steering strategy }\end{array}$ & $\begin{array}{l}\text { Steering actions are selected based on the orientation of the vehicle within a look-ahead time (a parameter } \\
\text { currently defined as } 1 \mathrm{~s} \text { ) as calculated in server F using the following logic: If the vehicle's orientation within the } \\
\text { look-ahead time is close to the center of the lane }( \pm 0.1 \mathrm{~m}) \text {, no action is taken. Otherwise, if it is within the lane } \\
\text { boundaries, a normal steering action is initiated, or if it is outside the lane boundaries, an imminent steering action } \\
\text { is initiated. }\end{array}$ \\
\hline Steering action & $\begin{array}{l}\text { A new steering angle is calculated at server } \mathrm{F} \text { and executed by servers } \mathrm{V}, \mathrm{W}, \mathrm{X} \text {, and } \mathrm{Y} \text { as a function of the } \\
\text { orientation at look-ahead time and the selected steering strategy (normal or imminent). Normal actions are } \\
\text { characterized by a steering movement to the new steering angle, and then back to a neutral position. Imminent } \\
\text { actions are characterized by a larger magnitude of steering angle and a shorter interval until the steering wheel is } \\
\text { returned to its neutral position. }\end{array}$ \\
\hline
\end{tabular}

\section{Interface with a Driving Simulator}

To provide an off-the-shelf vehicle dynamics module to the system and to examine the ability of the QN-MHP steering model to produce relevant steering actions in real-time, the steering model (implemented in ProModel) was interfaced with a fixed-based driving simulator (DriveSafety 500c). The simulator sends position information to the model. Whenever a glance is made to a specific position in the road scene, and certain information is assumed by the model to be available, it retrieves that information from the driving simulator. The model sends steering commands to the driving simulator after every hand movement is made. The driving simulator retrieves these steering commands continuously to steer the vehicle. (See Tsimhoni and Liu, 2003 for more details on the interface between Promodel and the driving simulator.)

\section{Empirical Validation}

As an initial empirical validation of the current steering model, data were compared to baseline driving data from a fixed-based driving simulator experiment (Tsimhoni and Green, 2003). In that experiment, 24 subjects participated, 12 younger (ages 20-28, mean of 23) and 12 older (ages 65-71, mean of 68). After driving a practice road, subjects drove on a $3.6 \mathrm{~m}$ wide, single-lane road consisting of a straight section and two curves $(\mathrm{r}=200 \mathrm{~m}$ and $\mathrm{r}=400 \mathrm{~m})$. This simulator experiment was chosen as the first test of the model because road geometry was simple and well controlled, there was no traffic, subjects were using cruise controlled speed, and only visual information was available to drivers, which matched the assumptions of the current steering model. Additionally, data from the experiment were readily available to the authors.

For the comparison, 8 simulation runs of the QN-MHP steering simulation model were run on a road that had the same geometry. Each run was based on a different random seed, to provide variability in the service times within the network. 


\section{RESULTS}

The model demonstrated realistic steering behavior. It steered a simulated vehicle within the lane boundaries on a straight section and on two curves. Four dependent measures of driving performance were used for a quantitative comparison of the model and the empirical data. Table 2 shows the driving parameters produced by the steering model in comparison to the same parameters from the validation experiment. Analysis of variance was performed for each of the four dependent measures using three levels of road curvature nested within two levels of model validation (simulated/empirical). Overall, differences between the models were small and the trends due to road curvature were similar.

Mean steering angle in the simulation was up to .3 degrees lower than in the empirical data, $F(1,28)=7.3, p<.05$, but the interaction between model-validation and curvature was not significant, $F(2,56)=3.3$. The SD of steering wheel angle in the simulation was about half a degree less than in the empirical data, $F(1,28)=5.1, p<.05$, but the interaction between model-validation and road was not significant, $F(2,56)=.3$. Thus, suggesting that there was less variability in the steering angle in the simulation model, but the increase in variability due to road curvature was the same as in the empirical data.

The difference between mean lateral position in the simulation and in the empirical data was not significant, $F(1,28)=.7$, and neither was the interaction with road curvature, $F(2,56)=1.9$. However, the mean lateral position in the simulation on the sharp curve was slightly higher than in the empirical data. Finally, the difference between SD of lateral position in the simulation and in the empirical data was not significant, $F(1,28)=.01$, but there was an interaction with road curvature, in which the SD on the sharp curve was higher in the simulation than in the empirical data, $F(2,56)=7.2$, $p<.01$. Thus, suggesting that the model was able to maintain lateral position but was less effective on the sharp curve.

Figure 2 illustrates the resulting driving performance of the model. The mean lateral position on a $400 \mathrm{~m}$ radius right curve is shown, as predicted by the model across 8 runs. The vehicle remains within the lane boundaries and there is more variability on the curve. Figure 3 illustrates driving performance of real subjects on the same road in the driving simulator. Several differences are noted: (1) the model is generally less consistent across time, (2) on curvature change, the model's timing is not as good as that of real subjects, and (3) the variability between runs is smaller than the variability between subjects.

Table 2. Driving performance parameters as predicted by the steering model and as measured in a driving simulator experiment.

\begin{tabular}{llcccccc}
\hline & \multicolumn{3}{c}{ Steering model } & \multicolumn{3}{c}{ Empirical validation } \\
\hline $\begin{array}{l}\text { Road (Curve Radius [m]) } \\
\text { Steering Angle [deg] }\end{array}$ & Straight & 400 & 200 & Straight & 400 & 200 \\
& & & & & & & \\
& Mean & $.01 \pm .02$ & $17.4 \pm .05$ & $34.8 \pm .6$ & $0 \pm 0$ & $17.5 \pm .6$ & $35.1 \pm .6$ \\
Lateral Position [m] & SD & $.36 \pm .04$ & $1.06 \pm .28$ & $3.3 \pm 1.5$ & $1.1 \pm .5$ & $1.7 \pm .6$ & $3.7 \pm 1.4$ \\
& Mean & $.01 \pm .03$ & $.12 \pm .10$ & $0.44 \pm .10$ & $.01+.22$ & $.13 \pm .20$ & $.27 \pm .22$ \\
& SD & $.07 \pm .01$ & $.11 \pm .06$ & $0.26 \pm .12$ & $.11 \pm .04$ & $.15 \pm .05$ & $.19 \pm .05$ \\
\hline
\end{tabular}

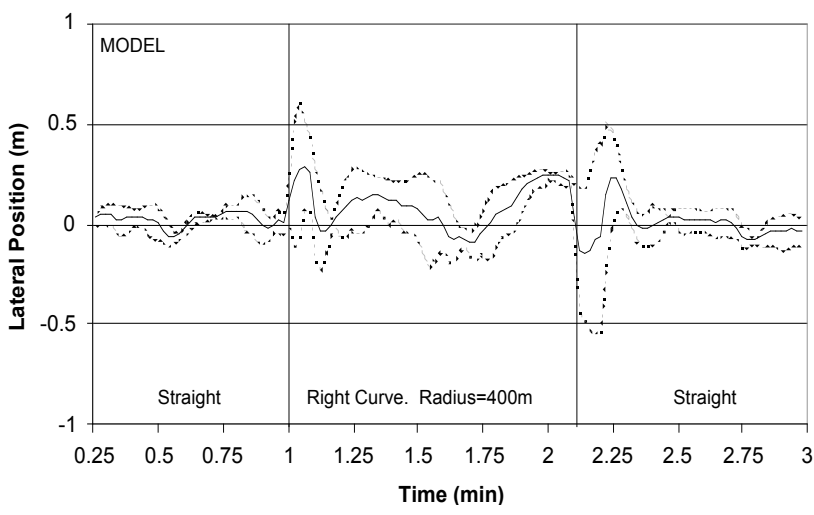

Figure 2. Lateral position as predicted by simulation model. Solid line - mean of 8 runs, dashed lines - range of \pm 1 SD between runs

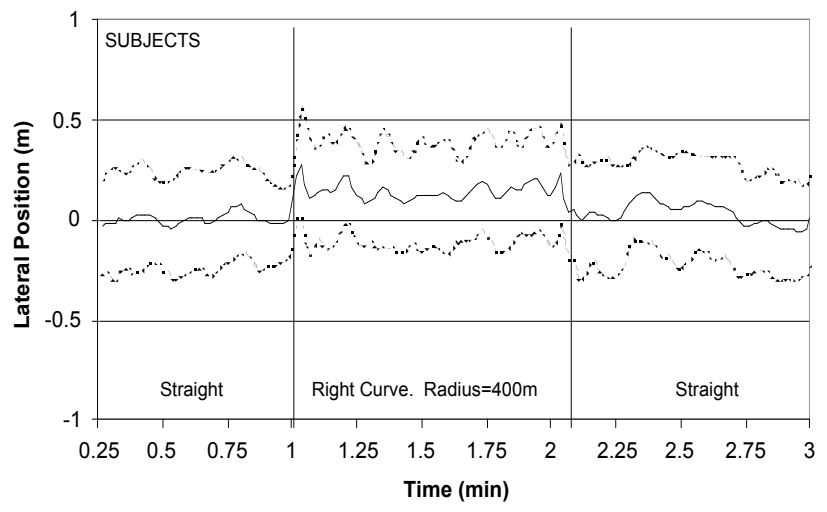

Figure 3. Lateral position as measured in driving simulator experiment with real subjects. Solid line - mean of 24 subjects, dashed lines - range of \pm 1 SD between subjects 


\section{DISCUSSION}

As mentioned earlier, the potential strength of QN-MHP as a model of driving behavior is in its ability to add concurrent activities without limiting or predefining their order of occurrence and to model human performance with an underlying, context-free, modeling architecture. The success of modeling concurrent perceptual, cognitive, and motor activities of steering in a truly concurrent, context-free, network architecture, as illustrated in the current study, opens the door for modeling other concurrent activities.

The successful interfacing of ProModel with a driving simulator opens a range of possible areas of research and application for the current model. For example, it allows for visible and real-time, demonstration of the steering strategy implemented in the model. It also provides for an off-theshelf dynamics module that can be manipulated independent of the driver model.

Our on-going research builds upon the current work and expands it in two aspects: (1) The driving task will be expanded to include speed control and to alter behavior based on traffic. (2) A secondary in-vehicle task will be added as a parallel activity. As other perceptual modalities are added to the QN-MHP architecture (e.g., vestibular, auditory), their addition to the driving task, and their effects on it, will be investigated.

\section{REFERENCES}

Anderson, J. R., \& Lebiere, C. (1998). The atomic components of thought. Hillsdale, NJ: Erlbaum.

Donges, E. (1978). A two-level model of driver steering behavior. Human Factors, 20 691-707

Feyen, R. G., \& Liu, Y. (2001a). Modeling task performance using the queuing network - model human processor (QN-MHP). Proceedings of the $4^{\text {th }}$ International Conference on Cognitive Modeling.

Feyen, R. G., \& Liu, Y. (2001b). The queuing network model human processor (QNMHP): An engineering approach for modeling cognitive performance. Proceedings of the Human Factors and Ergonomics Society 45th annual meeting.

Hildreth, E. C., Beusmans, J. M. H., Boer, E. R., \& Royden, C. S. (2000). From vision to action: Experiments and models of steering control during driving. Journal of Experimental Psychology: Human Perception and Performance, 26(3), 1106-1132.

Land, M. F., \& Lee, D. N. (1994). Where we look when we steer. Nature, 369, 742-744

Leibowitz, H. W., \& Owens, D. A. (1977). Nighttime driving accidents and selective visual degradation. Science, 197, 422-423.

Levison, W. H. (1989). The optimal control model for manual controlled systems. In G. R. McMillan et al., (Eds.) Applications of human performance models to system design, Plenum Press, New York, pp. 185-198.

Levison, W. H. (1993). A simulation model of Driver's use of in-vehicle information systems. Transportation Research Record 1403, pp. 7-13.
Levison, W. H., Simsek, O., Bittner, A. C., \& Hunn, S. J. (2001). Computational techniques used in the driver performance model of the interactive highway safety design model. Transportation Research Record 1779, pp. 17-25.

Loomis, J. M., \& Beal, A. C. (1998). Visually controlled locomotion: Its dependence on optic flow, three-dimensional space perception, and cognition. Ecological Psychology, 10(3,4), 271-285

McRuer, D. T., Allen, R. W., Weir, D. H., \& Klein, R. H. (1977). New results in driver steering control models. Human Factors, 19(4), 381397

Mourant, R. R., \& Rockwell, T. H. (1970). Mapping eye-movement patterns to the visual scene in driving: An exploratory study. $\underline{\text { Human }}$ Factors, 12, 81-87.

Owens, D. A., \& Tyrrell, R. A. (1999). Effects of luminance, blur, and age on nighttime visual guidance: A test of the selective degradation hypothesis. Journal of Experimental Psychology: Applied, 5, 115-128

Reymond G, Kemeny A, Droulez J, \& Berthoz A. (2001). Role of lateral acceleration in driving: experiments on a real vehicle and a driving simulator. Human Factors, 43(3), 483-495

Salvucci, D. D., \& Macuga, K. L. (2002). Predicting the effects of cellular-phone dialing on driver performance. Cognitive Systems Research, 3, 95-102.

Salvucci, D. D., Boer, E. R., \& Liu, A. (2001). Toward an integrated model of driver behavior in a cognitive architecture. Transportation Research Record, 1779.

Summala, H. (1998). Forced peripheral vision driving paradigm: Evidence for the hypothesis that car drivers learn to keep in lane with peripheral vision. In A.G. Gale et al. (Eds.), Vision in Vehicles VI (pp. 51-60). Amsterdam: Elsevier Science Publishers.

Tsimhoni, O., \& Green, P. (2003). Time-sharing of a visual in-vehicle task while driving: Effects of four key constructs. In D. V. McGehee, J. D. Lee, \& M. Rizzo (Eds.) Driving Assessment 2003: International Symposium on Human Factors in Driver Assessment, Training, and Vehicle Design.

Tsimhoni, O., \& Liu, Y. (2003). Steering a driving simulator using the Queueing network-model human processor (QN-MHP). In D. V. McGehee, J. D. Lee, \& M. Rizzo (Eds.) Driving Assessment 2003: International Symposium on Human Factors in Driver Assessment, Training, and Vehicle Design.

Wallis, G., Chatziastros, A., \& Bulthoff, H. (2002). An unexpected role for visual feedback in vehicle steering control. Current Biology, 12, 295-299.

Wann, J. P., \& Land, M. F. (2000). Steering with or without the flow: Is the recovery of heading necessary? Trends in Cognitive Science 4, 319 -324 . 